\title{
Detecção Cooperativa em Redes Cognitivas para o Controle da Interferência em Sistemas Licenciados
}

\author{
André Chaves Mendes e José Ferreira de Rezende
}

\begin{abstract}
Resumo-Em redes cognitivas, os dispositivos de uma rede oportunista aproveitam os momentos de ociosidade do sistema licenciado para realizar as suas transmissões. Para tal, é fundamental que esses períodos de ociosidade sejam detectados com confiabilidade. Diante desta necessidade, o presente trabalho propõe um mecanismo de deteç̧ão cooperativa dos sinais transmitidos pelo sistema licenciado, através da utilização de dispositivos sensores equipados com um sistema de localização, fornecendo informações para o controle da potência transmitida pelos dispositivos da rede oportunista de forma a limitar a interferência causada no sistema licenciado. As simulações realizadas mostram que num cenário ad hoc é possível melhorar a qualidade das comunicações da rede oportunista, mantendo a interferência dentro dos limites permitidos.
\end{abstract}

Abstract-In cognitive networks, the devices of a opportunistic network take advantage of the idle moments of the licensee to carry out their transmissions. Therefore, it is essential that this periods of idleness are detected with reliability. Given this need, this paper proposes a cooperative mechanism for detection of signals transmitted by the licensee, through the use of sensor devices equipped with a location system, providing information to control the power transmitted by the opportunistic network devices in order to limit the interference caused in the licensee. The simulations performed show that in an ad hoc scenario is possible to improve the quality of opportunistic network communications, keeping interference within the limits allowed.

\section{INTRODUÇÃO}

A crescente demanda por faixas do espectro de radiofrequências juntamente com o uso ineficiente das bandas licenciadas [10] trouxe à tona a possibilidade de se abrirem essas bandas de frequências subutilizadas para acesso dinâmico oportunístico [2]. Seguindo nesse caminho, as pesquisas sobre redes reconfiguráveis (fixas e móveis) têm se focado em dispositivos como o rádio cognitivo [6], evolução natural do rádio definido por software que combina aspectos de inteligência artificial e de radiocomunicações, capaz dentre outras coisas, de adaptar dinamicamente a potência das suas transmissões às condições do ambiente onde ele se encontra.

As redes cognitivas [14], ao contrário das demais redes (legadas), são capazes de adaptar o seu funcionamento (proativamente ou reativamente) em resposta à estímulos externos. Isto é conseguido através de mecanismos que captam esses estímulos do ambiente e obtêm conhecimento a partir desta interação, planejando, em conformidade, suas ações futuras.

Apesar dessa flexibilidade, não há certeza de que os rádios cognitivos, integrantes de uma rede cognitiva oportunista (ou secundária), funcionem sem causar interferência excessiva nos sistemas licenciados (ou primários). Essa certeza é essencial para que faixas de frequências sejam compartilhadas e

André Chaves Mendes e José Ferreira de Rezende, Grupo de Teleinformática e Automação (GTA), Programa de Engenharia Elétrica (PEE), COPPE/UFRJ, Rio de Janeiro, Brasil, E-mails: \{andre, rezende\}@ gta.ufrj.br regulamentadas para o uso por sistemas secundários, como está sendo feito para a banda de TV analógica nos EUA, para a qual o FCC (Federal Communications Commision) estabeleceu regras de uso a partir de 2009 [3]. Também não há consenso de que redes sem fio distintas, compartilhando a mesma faixa de frequências, funcionem adequadamente sob forte controle da sua potência de transmissão e da interferência que causam nas demais redes e sistemas.

Essa problemática envolvendo interferência entre dispositivos primários e secundários tem recebido grande atenção ultimamente [7], [8], [9], [21], [22], [23]. Entretanto, em todas essas propostas, a abordagem foi feita através de uma análise teórica e probabilística, se baseando num modelo de rede primária com um transmissor e múltiplos receptores passivos.

Variando um pouco desse modelo de rede primária, propomos uma rede secundária ad hoc com nós FIXOS e MÓVEIS, composta por rádios cognitivos, que efetuam a detecção cooperativa das transmissões da rede primária e utilizam essa informação para ajustar sua potência de transmissão, mantendo a conectividade entre si e controlando dinamicamente a interferência percebida pela rede primária. A conectividade, no caso, é encarada como um indicador simples da qualidade nas comunicações.

No restante deste artigo, a seção seguinte dá uma visão geral sobre os conceitos básicos necessários à compreensão do artigo e descreve os elementos componentes das redes primária e secundária, bem como as considerações feitas. A seção III quantifica os parâmetros usados, descreve os cenários empregados na proposta e mostra os resultados das simulações. Finalmente, a seção IV conclui o artigo e cita trabalhos futuros.

\section{Conceitos E DescriçÃo do Sistema}

O sistema proposto é composto por uma rede secundária $a d$ hoc com nós fixos e móveis, composta por rádios cognitivos, que efetuam a detecção cooperativa das transmissões da rede primária e utilizam esta informação para ajustar sua potência de transmissão, mantendo a conectividade entre si e controlando dinamicamente a interferência causada na rede primária.

A rede primária é a rede licenciada para utilizar a banda de frequência de interesse. A rede secundária funciona de maneira oportunista, evitando ultrapassar o limite de interferência tolerado pela rede primária, que é previamente divulgado.

Em ambas as redes, todos os nós são transceptores que não utilizam técnicas de espalhamento espectral, podendo trocar dados somente na mesma banda de frequência (banda de interesse), e fazem uso de um mecanismo de reserva do meio semelhante ao RTS/CTS do IEEE 802.11b visando reduzir o problema do terminal escondido, mantendo todos os demais nós dentro do alcance cientes da comunicação em andamento. 
Não há um canal de controle estabelecido, entretanto, há um controle distribuído sendo exercido na rede secundária através da troca de mensagens. Segue-se uma descrição das redes.

Rede Primária. Os nós que a compõem (nós primários, P) são capazes apenas de comunicarem entre si e possuem alcance de transmissão fixo (alcance padrão), que é usado como valor de referência para os demais nós. Durante o período em que efetuam alguma transmissão (janela de localização), os nós Ps poderão ser detectados e localizados pelos nós sensores da rede secundária.

O limite de interferência permitido pelos nós primários é divulgado para a rede secundária e vale $I$. O valor, a priori, é referenciado à posição do nó, mas poderia ser calculado para uma outra posição considerando a existência de círculos de nível de sinal interferente permissíveis crescentes com a distância (Figura 1).

Rede Secundária. Emprega rádios cognitivos e é composta por nós desempenhando distintamente a função operacional, responsável pela comunicação na rede, e a função sensorial, responsável pela detecção cooperativa das transmissões dos nós primários e pela obtenção de uma estimativa da distância e da direção de chegada do sinal detectado. Desta forma, chamaremos de nó sensor, $\mathrm{S}$, o nó da rede secundária desempenhando a função sensorial; e de nó operacional, O, o nó da rede secundária desempenhando a função operacional.

Para reduzirmos os problemas causados pelos efeitos de multi-percurso e sombreamento, que fazem com que o sensoriamento individual não seja adequado para a detecção dos usuários primários com precisão [15], e melhorarmos a precisão na detecção dos usuários primários, implementamos nos nós sensores o sensoreamento cooperativo.

O conhecimento pelos nós da rede secundária da sua posição na área onde atuam é de grande importância para o funcionamento da rede. Para a escolha do sistema de posicionamento a ser adotado, vários requisitos como escalabilidade, disponibilidade, confiabilidade, eficiência energética e precisão, são fatores importantes. Apesar de não o implementarmos de fato, pressupomos que todos os dispositivos da rede secundária possuem um sistema de posicionamento que fornece informações acerca da sua posição na região onde se encontram.

Os nós sensores permanecem a maior parte do tempo sem transmitir (modo passivo) empenhados em efetuar a detecção das transmissões dos nós primários e obter uma estimativa da distância e da direção de chegada do sinal detectado. Além disso, eles possuem dois mecanismos que são:

- Mecanismo de detecção de transmissão. A detecção de sinais possui variada aplicação na Ciência, inclusive nas comunicações. Em [20] encontramos uma coletânea dos resultados e técnicas de detecção de sinais desenvolvidos nas últimas décadas. Desde então, alguns métodos foram propostos com essa finalidade: detecção por energia [18], detecção pela forma de onda do sinal [4], detecção pela cicloestacionariedade do sinal [18], detecção por filtros casados [1], etc.. Todos possuem vantagens e desvantagens e se aplicam melhor ou pior de acordo com as características do sinal a ser detectado. Face a facilidade de implementação, pressupomos que o nó sensor pode detectar, dentro do seu alcance, qualquer transmissão dos demais nós fazendo uso do método de detecção por energia.

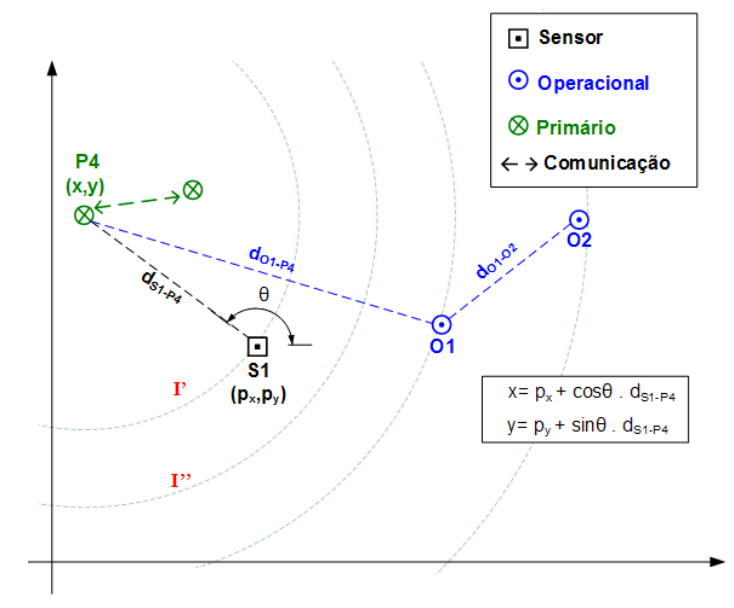

Fig. 1. Círculos de interferência e mecanismo de localização do nó primário.

Se o nó sensor considerar que a fonte do sinal detectado não é um nó da rede secundária, ele envia uma mensagem para os nós operacionais dentro do seu alcance com o objetivo de alertar sobre a presença de um nó primário transmissor e inicia o procedimento de localização desse nó através do mecanismo de localização do primário. Uma vez localizado o nó primário que está transmitindo, o nó sensor envia outra mensagem para os nós operacionais dentro do seu alcance, contendo a posição estimada do nó primário transmissor, e volta a operar passivamente até que aconteça outra detecção. - Mecanismo de localização do primário. É iniciado após a detecção da transmissão e sua identificação negativa como nó da rede secundária.

O requisito básico de qualquer sistema de localização é permitir a correta medição da distância entre quaisquer dois nós da rede. Várias propostas que foram feitas visando a determinação dessa distância incluíram os métodos de Timeof-Arrival (ToA), Time Difference of Arrival (TDoA), Angleof-Arrival (AoA) e Received-Signal-Strength (RSS) [5], [17]. As principais fontes de erros nesses sistemas são provenientes da visada indireta entre os dispositivos (NLOS) e da medição propriamente dita. Métodos para detectar e corrigir erros devido à NLOS são apresentados em [11], [13].

De um modo geral, as medições de distâncias sofrem por erros cuja variação é dependente do tempo, ou então, por erros estáticos, mas dependentes do ambiente. Os erros com variação temporal causados, por exemplo, por ruído aditivo e interferência, podem ser reduzidos realizando múltiplas medições numa janela de tempo e calculando a média dessas medições. Já os erros estáticos dependentes do ambiente são resultado, principalmente, da disposição dos obstáculos na região de operação. Uma vez que a região de operação normalmente não é a mesma, esse tipo de erro torna-se imprevisível e deve ser modelado como uma variável aleatória.

O escopo do artigo não inclui uma descrição dos algoritmos de localização, entretanto, assumimos que o método AoA é utilizado pelo nó sensor para obter uma linha de posição sobre a qual estará o nó primário transmissor. Também é utilizado o método RSS para obter uma estimativa da sua distância ao mesmo nó primário. 
A posição (x,y) do nó primário detectado é obtida conforme a Figura 1 e informada para os nós operacionais no alcance através de uma mensagem. A precisão da posição estimada do nó transmissor e da sua distância dependerá exclusivamente do número de nós sensores que efetuarem a detecção. No caso de ser detectada mais de uma transmissão simultânea distinta pelo mesmo nó sensor (mais de um nó detectado) ${ }^{1}$, a janela de localização é alongada.

Os nós operacionais são capazes de comunicarem apenas entre si (ponto-a-ponto) de forma semelhante aos nós primários e compartilham a banda de interesse, preferencialmente quando não houver nenhum nó primário fazendo uso dela, ou simultaneamente a eles, mas de modo a nunca superar o limite de interferência $I$. Para isso, recebem informações dos nós sensores para realizar o controle de sua potência de transmissão. O seu alcance de transmissão é ajustável entre zero, correspondente a não efetuar nenhuma transmissão, e o alcance padrão, correspondente a se utilizar a máxima potência de transmissão.

Ao tomar conhecimento da posição estimada do nó primário transmissor, o nó operacional promove (ou não) o ajuste da sua potência de transmissão, usando as informações contidas na mensagem oriunda do nó sensor que promoveu a detecção e localização.

De forma a validar o sistema proposto, efetuamos algumas considerações necessárias ao funcionamento das redes que são descritas a seguir:

- Em todas as mensagens trocadas na rede secundária, há informações de identificação do nó origem e do nó destino, sua posição e sua potência de transmissão;

- Todos os nós sensores possuem receptores com sensibilidade $10 \mathrm{~dB}$ acima da base de ruído (noise floor) presente na banda de interesse;

- Todos os nós sensores possuem um par de antenas: uma para comunicação e outra (feixe de antenas direcionais) para efetuar a detecção de transmissões primárias (AoA);

- Na área onde as redes estão operando, não existem emissões de radiofrequência capazes de introduzir erros significativos nos mecanismos propostos;

- As comunicações se darão sem erros. A existência de erros causaria a retransmissão de pacotes, dado que não foi considerado para as métricas avaliadas nesta etapa da avaliação.

\section{Simulação: DescriçÃo E Resultados}

Para avaliar o comportamento da rede cognitiva proposta, construímos um simulador usando a ferramenta Matlab [12]. O simulador foi dividido em blocos e o seu fluxograma pode ser visto na Figura 2.

Criamos quatro cenários, três FIXOS e um MÓVEL, para avaliarmos o comportamento das redes primária e secundária (Figura 3).

Nos cenários FIXOS, inicialmente distribuímos aleatoriamente os nós sensores na área e logo após os demais nós, de forma que os sinais transmitidos tanto pelos nós primários

\footnotetext{
${ }^{1}$ A detecção distinta do sinal está diretamente ligada a capacidade de separação espacial dos sinais detectados referente ao feixe de sensores que compõem a antena direcional utilizada na detecção.
}

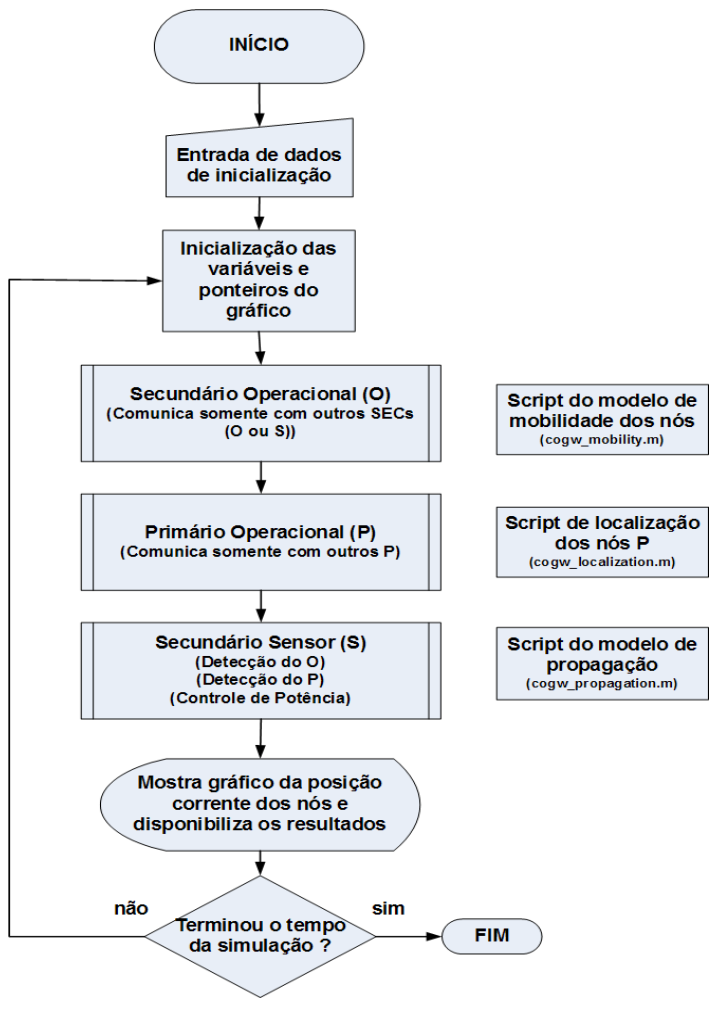

Fig. 2. Fluxograma do simulador construído usando o Matlab.

quanto pelos nós operacionais possam alcançar os nós sensores. A posição dos nós em ambas as redes é importante sendo desejável que não haja uma total intersecção entre as redes primária e secundária, pois caso contrário haverá uma paralisação das transmissões na rede secundária a fim de se manter o limite de interferência $I$. Apesar disso, nenhum controle nesse aspecto é realizado. Os nós primários estão sempre dentro do alcance um do outro. Os nós operacionais inicialmente também estão. Os cenários são:

- Cenário 1: 2 nós operacionais (O1 e O2), 2 nós primários (P1 e P2) e 2 nós sensores (S1 e S2);

- Cenário 2: 4 nós operacionais (O1 a O4), 2 nós primários (P1 e P2) e 2 nós sensores (S1 e S2);

- Cenário 3: 4 nós operacionais (O1 a O4), 4 nós primários (P1 a P4) e 2 nós sensores (S1 e S2).

O valor da potência de transmissão inicial dos nós operacionais é sorteado entre os valores cujos alcances atingem $80 \%$ e $100 \%$ do alcance padrão. Os nós primários comunicantes usam o valor máximo da sua potência de transmissão, correspondente ao alcance padrão.

O sinal é recebido na posição do nó sensor como $p r$, já atenuado conforme o modelo de propagação log-normal shadowing [16]. Assim, nos nós sensores temos que garantir que $\left(\sum p r_{O} \leq I^{\prime}\right.$ ) (Figura 1) para não causar interferência acima do permitido nos nós primários.

Acompanhando pela Figura 3, inicialmente, o nó S1 faz a detecção antes de S2 e detecta primeiro O1 (e O3) e depois P1 (e P3). Devemos ressaltar que, nessa situação, não ocorre transmissão simultânea dos nós operacionais por conta do uso do mecanismo de reserva do meio. Dependendo do cenário, o 


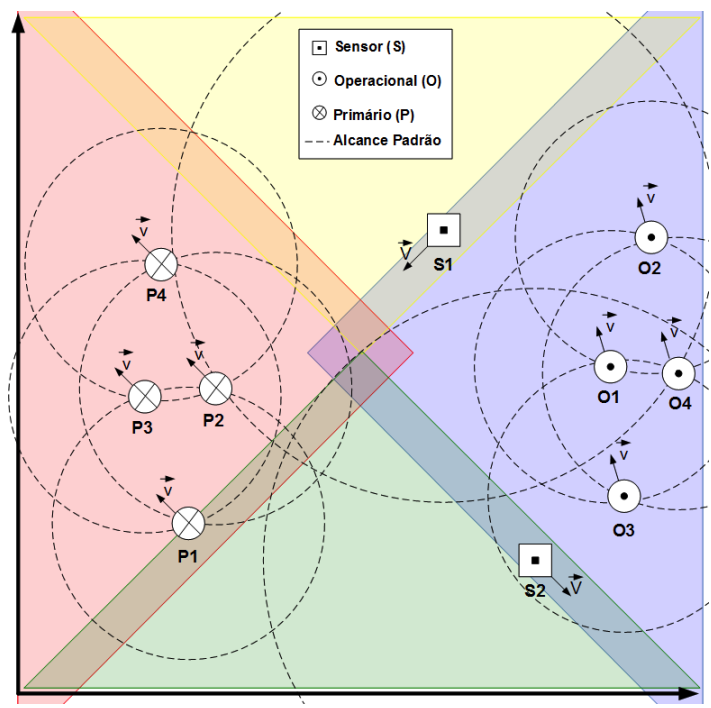

Fig. 3. Esquema de posicionamento e mobilidade para os cenários FIXOS e MÓVEL.

nó $\mathrm{S} 1$ efetuará os cálculos abaixo onde variável $\eta$ representa a base de ruído (noise floor):

$$
S I N R_{O 1} \approx \frac{p r_{O 1}}{\eta}, S I N R_{O 3} \approx \frac{p r_{O 3}}{\eta}
$$

(Sem interferência entre os nós Ps)

$$
S I N R_{P 1} \approx \frac{p r_{P 1}}{p r_{(\text {O1 ou } 03)}+\eta}, S_{N N R_{P 3}} \approx \frac{p r_{P 3}}{p r_{(\text {O1 ou } O 3)}+\eta}
$$

Caso haja uma colisão no receptor de S1, por transmissões simultâneas de nós primários e secundários ou por conta de RTS/CTS, a medida é descartada e aguarda-se nova detecção.

No cenário MÓVEL (4), composto por 4 nós operacionais (O1 a O4), 4 nós primários (P1 a P4) e 2 nós sensores (S1 e $\mathrm{S} 2$ ), a área quadrada disponível é dividida em quatro setores pelas diagonais do quadrado. Em setores opostos, com uma ligeira superposição próxima do centro do quadrado, ficam os nós primários e os nós operacionais (Figura 3), que dentro dos respectivos setores seguem o modelo de mobilidade de grupo baseado no Reference Point Group Mobility (RPGM).

O movimento dos nós sensores segue o modelo de mobilidade Fixed Waypoint (FWP) e acontece em movimentos opostos sobre as duas semi-diagonais do quadrado que forma o setor onde eles se encontram, que limitam a região onde se movimentam os nós operacionais. Ambos os modelos de mobilidade estão descritos em [19].

Neste cenário, S1 e S2 podem detectar as transmissões dos nós $\mathrm{O} 1$ a $\mathrm{O} 4$, e dos nós $\mathrm{P} 1$ a $\mathrm{P} 4$, mas não a todo momento, dada a mobilidade. O valor da potência de transmissão inicial dos nós é escolhido da mesma forma que nos cenários FIXOS.

O sinal é recebido na posição do nó sensor como $p r$, já atenuado conforme o modelo de propagação. Assim, no nó sensor temos que garantir que $\left(\sum p r_{O} \leq I^{\prime}\right)$ num determinado instante de tempo (snapshot).

Da mesma forma que nos cenários FIXOS, a abordagem do problema é feita considerando que caso haja uma colisão no receptor de $\mathrm{S} 1$, a medida é descartada e aguarda-se nova detecção. Como a velocidade do nó é baixa, para efeito de cálculo, consideramos o círculo de reserva do meio centrado nos nós ${ }^{2}$.

As medidas feitas pelos nós sensores são as mesmas dos cenários FIXOS, porém o acréscimo da mobilidade traz complicadores, principalmente, para o algoritmo de localização do primário, que precisa estabelecer uma linha de posição num intervalo de tempo curto (janela de localização $\leq$ snapshot).

Para a simulação, utilizamos uma área quadrada sem obstáculos de lado igual a $D$ onde dispomos os nós para cada um dos cenários. As variáveis usadas, bem como os valores que elas assumem estão resumidos na Tabela I.

\begin{tabular}{|c|c|l|}
\hline Nome & Valor & Conteúdo \\
\hline \hline$D$ & $100 \mathrm{~m}-3000 \mathrm{~m}$ & lado da área quadrada \\
\hline$f$ & $1 \mathrm{GHz}$ & frequência utilizada \\
\hline$\eta$ & $-100.9 \mathrm{dBm}$ & noise floor \\
\hline$X$ & $N\left(0 ; 5^{2}\right)$ & log distance path loss \\
\hline$Y$ & $N\left(0.01 d ; 0.05 d^{2}\right)$ & erro em distância (RSS) \\
\hline$\epsilon$ & $1 \%$ & erro no posicionamento \\
\hline$\phi$ & $1 \%$ & erro no AoA \\
\hline
\end{tabular}

TABELA I

VARIÁVEIS USADAS.

Para efeitos de cálculo, consideramos que o meio de transmissão é a atmosfera, ao nível do mar, com índice de refração unitário e que a velocidade de propagação das ondas de RF nesse meio é igual à velocidade de propagação da luz no vácuo. A potência de transmissão (EIRP) vale $0 \mathrm{dBm}$ e corresponde ao alcance padrão.

Representamos os efeitos de sombreamento no modelo de propagação log-normal shadowing como uma variável aleatória normal $X \sim N\left(\mu, \sigma^{2}\right)$. O tempo máximo da troca de mensagens (RTT) é da ordem de algumas centenas de $\mu$ segundos, considerando a maior distância entre os nós.

Em relação à localização dos nós primários transceptores, consideramos um erro único na estimação da sua distância $d$, que contabiliza os erros variáveis e os estáticos dependentes do cenário, modelado como uma variável aleatória normal $Y \sim$ $N\left(\mu, \sigma^{2}\right)$ [17]. O erro na determinação do ângulo de chegada $(\phi)$ e o erro devido ao sistema de posicionamento $(\epsilon)$ são fixos e foram retirados da compilação dos artigos: [5], [11], [13].

No cenário MÓVEL, os nós possuem velocidades máximas de $1,8 \mathrm{~m} / \mathrm{s}$, equivalentes a uma caminhada comum. Com isso, o deslocamento do nó durante o tempo da troca de mensagens mais o tempo gasto pelo algoritmo de localização para conseguir estimar a posição é de no máximo $6,5 \mathrm{~m}$.

Consideramos uma interferência sobre um nó primário quando o valor do nível de interferência $I$ sobre ele é ultrapassado. Como forma de ajuste para o mecanismo de controle de potência, usamos em todos os cenários os valores $10 \%$ e $20 \%$ para o fator de ajuste ${ }^{3} \delta$ no cálculo de $I^{\prime}$ (limite de interferência projetado sobre a posição do nó sensor).

\footnotetext{
${ }^{2} \mathrm{Na}$ verdade, como os nós estão se movendo, a reserva do meio não consegue assumir uma forma circular centrada neles. Ela pode assumir qualquer forma.

${ }^{3} \mathrm{O}$ uso do fator de ajuste tem como objetivo minimizar os efeitos dos erros de detecção de transmissão dos nós primários, bem como os efeitos de colisão nos nós sensores entre pacotes oriundos dos nós operacionais e primários, que podem ser observados, para o cenário 1 (Figura 4). Esses erros não são determinísticos, uma vez que suas causas também não são.
} 

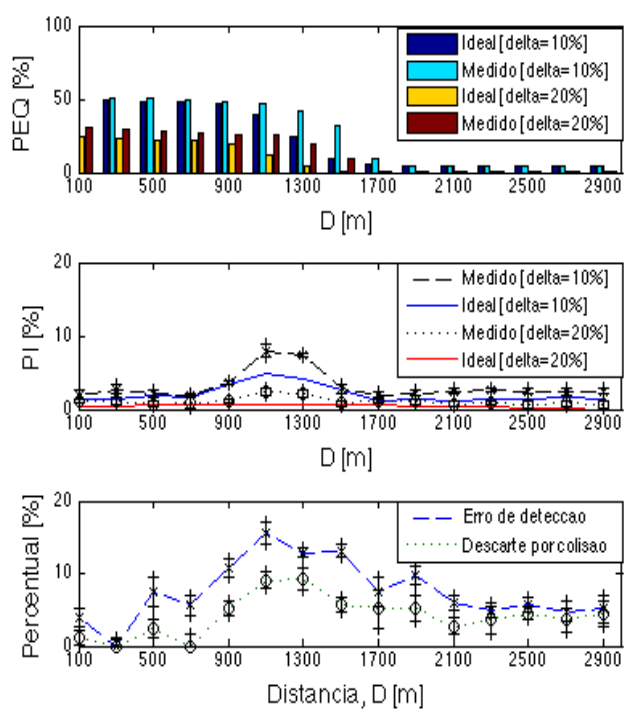

Fig. 4. Cenário 1.

Usamos o valor de $I$ equivalente a $-90 \mathrm{dBm}$ semelhante ao utilizado como limite de detecção da portadora nas interfaces comerciais que seguem o padrão IEEE 802.11b. O valor de I utilizado refere-se ao limite (fixo) de interferência tolerado pelos nós primários, que é divulgado no início da operação da rede primária.

Nossa intenção é mostrar que é possível que uma rede secundária funcione sem interferir prejudicialmente na rede licenciada, desde que essa divulgue o limite de interferência permitido na posição do seu transceptor. Para tanto, avaliamos individualmente em cada cenário o percentual de interferência $(P I)$ maior que $I$ no transceptor primário dada uma comunicação entre nós operacionais.

Queremos mostrar também que podemos manter a qualidade das comunicações na rede secundária mesmo sob restrição da interferência por ela causada avaliando a conectividade dos nós operacionais. Nesse caso, avaliamos o percentual de enlaces quebrados ( $P E Q$ ) na rede secundária por conta do controle de potência realizado pelo nó operacional.

Como comparação, criamos uma entidade externa à rede que possui todas as informações da sua dinâmica ao longo do tempo, porém sem os erros de posicionamento, de localização e de detecção do primário transmissor. Essa entidade fornece os valores ideais das métricas avaliadas.

Para observarmos o comportamento dos nós variamos a área quadrada disponível modificando a distância $D$, correspondente ao comprimento do lado do quadrado. Para a simulação, variamos o valor de D de $100 \mathrm{~m}$ a $3000 \mathrm{~m}$, de $100 \mathrm{~m}$ em $100 \mathrm{~m}$ e para cada cenário, fizemos 30 rodadas da simulação para cada valor de $D$ escolhido totalizando 900 rodadas.

O tráfego de dados na rede primária é modelado como uma fonte ON/OFF exponencial, com média do período ON e OFF, iguais, respectivamente, a $700 \mathrm{~ms}$ e $300 \mathrm{~ms}$. Os dados são gerados a taxa de $64 \mathrm{kbps}$ durante o período ON. De posse dessas informações passamos a analisar os resultados.
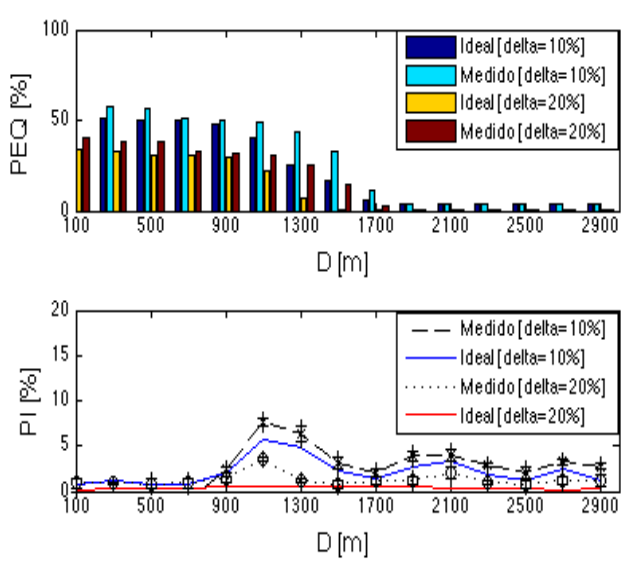

Fig. 5. Cenário 2.
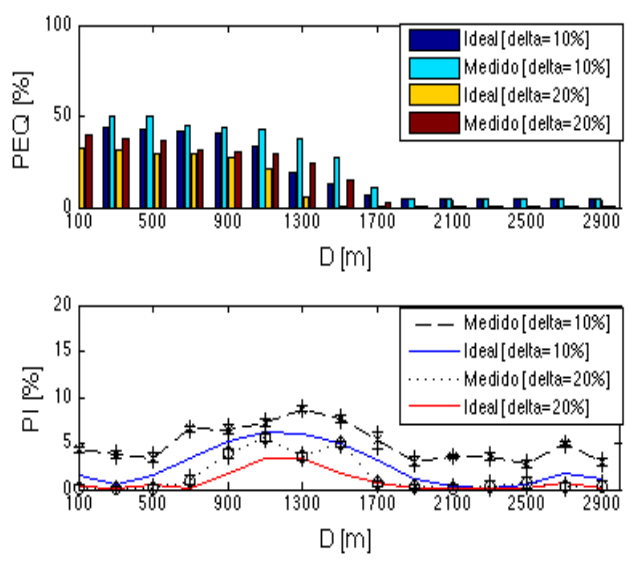

Fig. 6. Cenário 3.

Para $\delta=10 \%$, no cenário FIXO 1 (Figura 4), podemos notar que o PI medido é sempre maior que zero sobre o nó primário, embora para $D$ entre $100 \mathrm{~m}-800 \mathrm{~m}$ e para $D$ entre $1600 \mathrm{~m}-3000 \mathrm{~m}$, o valor de PI seja baixo, menor que $4 \%$.

Para $D$ entre $800 \mathrm{~m}$ e $1600 \mathrm{~m}$, o valor de PI medido possui um pico que assume valores de até $9 \%$. Analisando as causas desse crescimento, vemos que existe nos nós sensores um número elevado de colisões das mensagens transmitidas simultaneamente pelos nós operacional e primário nos mesmos intervalos de valores de $D$. Isso faz com que os nós sensores descartem as medições feitas, ocasionando o crescimento do valor de PI.

Outra causa para esse problema é a janela de tempo necessária para o mecanismo de localização obter a posição estimada do nó primário transmissor. Durante esse período, os nós operacionais continuam transmitindo, causando interferência. Somando-se a isso, os efeitos de desvanecimento do sinal dos nós primários com o aumento da distância dificultam a sua detecção precisa.

Um comportamento semelhante ao do cenário FIXO 1 pode ser observado nos demais cenários FIXOS (Figuras 5, 6 e 7) e a explicação dada pode ser estendida a eles..

No cenário MÓVEL (Figura 7), percebemos que não acon- 

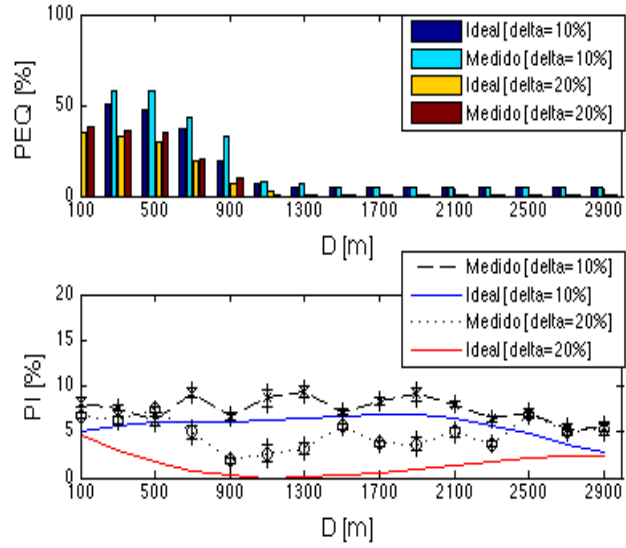

Fig. 7. Cenário 4

tece o efeito de crescimento no valor do PI medido, como nos cenários FIXOS, devido à variação da posição dos nós.

A velocidade baixa dos nós e a delimitação de regiões bem definidas para a sua movimentação, com pouca interseção entre elas, contribui para que o valor de PI se mantenha em até $10 \%$ durante toda a simulação.

Em relação ao valor do $P E Q$ medido na rede secundária (Figuras 4, 5, 6 e 7), vemos que em todos os cenários os valores são altos para valores pequenos de $D$ e decaem a medida que $D$ aumenta.

Uma explicação para esse comportamento é que por conta da pouca distância entre os nós e dos poucos intervalos de silêncio nos nós primários, há muita necessidade de controle da potência de transmissão nos nós secundários, o que ocasiona um valor alto para o PEQ. Mesmo nessa condição desfavorável, é possível manter a conectividade dos enlaces (100\%-PEQ) em cerca de $40 \%$ a $50 \%$, de acordo com o cenário.

Após um determinado valor de $D$, por conta dos efeitos de propagação, os sinais têm um alcance menor, tornando o controle de potência menos frequente e causando um decaimento mais forte da curva do PEQ medido.

Aumentando $\delta$ para $20 \%$ e observando, primeiramente, os cenários FIXOS, notamos que o PI medido é aproximadamente zero, assumindo valores de até $6 \%$ apenas para os valores de $D$ entre $800 \mathrm{~m}$ e $1600 \mathrm{~m}$, onde há maior incidência de erros de detecção e colisões. $\mathrm{O}$ valor do PEQ medido também apresenta uma redução e, consequentemente, a conectividade dos enlaces (100\%-PEQ) aumenta para $60 \%$ a $70 \%$, de acordo com o cenário. No cenário MÓVEL, observamos que o PI medido se mantém abaixo de $7 \%$ para toda a faixa de valores de $D$ e a conectividade fica em $60 \%$.

\section{CONCLUSÕES}

Por conta do esforço para a convergência de redes e serviços, da necessidade de reuso do espectro e da crescente abrangência e popularização do acesso à banda larga entre outros fatores, imaginamos que é questão de pouco tempo para que tenhamos, de fato, redes secundárias maciçamente em operação.
Variando um pouco do modelo de rede primária onde existe um transmissor com múltiplos receptores passivos, nossos resultados, com duas redes ad hoc e nós FIXOS e MÓVEIS, mostram que podemos implementar um controle (simples) da interferência sobre os nós primários apenas com um mecanismo de posicionamento individual e um sistema de detecção de fonte de sinal (radiogoniômetro). E simultaneamente, manter um nível aceitável de conectividade entre os nós da rede secundária, um indicador simples da qualidade nas comunicações.

Faz parte de nossos trabalhos futuros ajustar o mecanismo de localização/posicionamento para maior eficácia, finalizar a implementação do mecanismo para redução das colisões e estabelecer outras métricas e cenários mais "povoados" e avaliá-los.

\section{REFERÊNCIAS}

[1] D. Middleton. On the detection of stochastic signals in additive normal noise. IEEE Transaction on Information Theory, June 1957.

[2] FCC. FCC-03-322 - NOTICE OF PROPOSED RULE MAKING AND ORDER. Technical report, Federal Communications Commission, 30 December 2003

[3] FCC. FCC-08-260A1 - SECOND REPORT AND ORDER. Technical report, Federal Communications Commission, 14 November 2008.

[4] H. Tang. Some physical layer issues of wide-band cognitive radio systems. In IEEE Symposium on New Frontiers in Dynamic Spectrum Access Networks, November 2005.

[5] G. L. Stuber J. Caffery. Overview of radiolocation CDMA cellular systems. IEEE Communications Magazine, 36(4), April 1998.

[6] G. Q. Maguire Jr. J. Mitola III. Cognitive radio: making software radio more personal. IEEE Pers. Communications, 6(4):13-18, August 1999.

[7] K. B. Letaief K. Hamdi, W. Zhang. Power control in cognitive radio systems based on spectrum sensing side information. In IEEE Intern. Conference on Communications, 2007.

[8] E. Hossain L. Le. Osa-mac: A mac protocol for opportunistic spectrum access in cognitive radio networks. In IEEE Wireless Communications and Networking Conference, 2008.

[9] E. Hossain L. Le. Resource allocation for spectrum underlay in cognitive radio networks. In IEEE Trans. on Wireless Communications, 2008.

[10] M. A. McHenry. NSF Spectrum Occupancy Measurements Project Summary. Technical report, Shared Spectrum Company report, 2005.

[11] J. Holtzman M. P. Wylie. The N-LOS problem in mobile location estimation. In International Conf. on Univ. Pers. Communications, 1996.

[12] Inc. MathWorks. MATLAB - The Language Of Technical Computing. http://www.mathworks.com/products/matlab/.

[13] P.-C. Chen. A N-LOS error mitigation algorithm. In IEEE WCNC, 1999.

[14] J. Strassner P. Demestichas, G. Dimitrakopoulos. Introducing reconfigurability and cognitive networks concepts in the wireless world. In IEEE Vehicular Tech. Magazine, 2006.

[15] A. Sahai R. Tandra. Fundamental limits on detection in low SNR under noise uncertainty. Intern. Conf. on Wireless Networks, Communications and Mobile Computing, 2005.

[16] Theodore S. Rappaport. Wireless Communications: Principles and Practice. P. Hall, Inc., 2001.

[17] S. Gezici. A survey on wireless position estimation. Wireless Personal Comm., 2 October 2007.

[18] K. Challapali S. Shankar, C. Cordeiro. Spectrum agile radio: utilization and sensing architectures. In IEEE Symposium on New Frontiers in DSA Networks, November 2005.

[19] J. Boleng and V. Davies T. Camp. A survey of mobility models for ad hoc network research. 10 September 2002.

[20] V. Poor T. Kailath. Detection of stochastic processes. IEEE Trans. on Inf. Theory, October 1998.

[21] T. Peng e W. Wang W. Wang. Optimal power control under interference temperature constraints in cognitive radio network. In IEEE Wireless Comm. and Networking Conference, 2007.

[22] C. N. Mathur et all Y. Xing. Dynamic spectrum access with QoS and interference temperature constraints. In IEEE Trans. on Mob. Computing, 2007.

[23] Q. Zhao. Spectrum opportunity and interference constraint in opportunistic spectrum access. In IEEE Intern. Conference on Acoustics, Speech and Signal Processing, 2007. 